\title{
LXV. On the corpuscular theory of the thermoelectric forces
}

\section{Jakob Kunz Dr.phil.}

To cite this article: Jakob Kunz Dr.phil. (1908) LXV. On the corpuscular theory of the thermoelectric forces, Philosophical Magazine Series 6, 16:95, 767-789, DOI: 10.1080/14786441108636555

To link to this article: http://dx.doi.org/10.1080/14786441108636555

册 Published online: 21 Apr 2009.

Submit your article to this journal $\lceil\pi$

Џ Article views: 2

Q View related articles $\square$

Citing articles: 1 View citing articles 5 
platinum in different gases are atoms or molecules of the gas and not positive electrons out of the metal.

It is possible that the positive ionization in a vacuum is due to a real constituent which is common to the different elements examined, as opposed to some foreign substance whose presence is accidental. In this case it would be necessary to assume that the ions carry a charge which is smaller than the (at present) fundamental electronic charge $e$. This would follow from the fact that the value of $m / H$ for these ions is greater than the atomic weight of carbon, one of the substances from which they are produced. There is no other evidence, as yet, of the existence of fractions of this natural unit of electric charge.

It is proposed to extend the list of elements included in the investigation, and to institute other experiments which will decide between the preceding alternatives.

In conclusion the author wishes to thank Mr. P. Thomas, Instructor in the Laboratory, for his assistance during part of the investigation, and also Mr. F. Fisher, University Mechanician, for the care which he bestowed on the construction of the apparatus shown in fig. 1.

Princeton, N.J.

22nd June, 1908.

LXV. On the Corpuscular Theory of the Thernoelectrie Forees. By Jakob KUnz, Dr. phil., Privatdozent für Physik am eidg. Polytechnikum in Zürich *

\section{Contents.}

$\$ 1$. Potential-Differences between Metals in Contact.

2. Thermoelectric Force in unequally heated Metallic bar.

3. The Electromotive Force of Thermoelements.

4. Peltier effect.

5. Thomson effect.

6. Connexion between the different effects.

$\$ 7$. On the Electric Force X and Pyroelectricity.

$\$ 8$. Conservation of Energy.

9. Resistance of Alloys.

THE theoretical laws of the thermoelectric phenomena 1 have been derived from two different theories. The electromotive force, the Peltier effect, and the Thomson effect are related to one another by thermodynamic formulas, which were given first by Clausius $\dagger$ and Lord Kelvin + .

* Communicated by the Author.

+ Clausius, Pogg. Ann. xc. p. 513 (1853).

† W. Thomson, Edinburgh Roy. Soc. 'Transactions,' xxi. (1854). 
Lord Kelvin even predicted the last of the three phenomena, showing that in some metals an electric current carries heat from the hot to the cold parts of the metal, while in other metals the transference of the heat is in the opposite direction. The experiments proved in a very satisfactory way the existence of this effect first derived from theoretical considerations.

After an interval of about half a century, the corpuscular theory of metallic conduction has been applied to the thermoelectric phenomena by Riecke*, Drude $\uparrow, H$. A. Lorentz $\ddagger$, and J. J. Thomson $\S$. The results of the kinetic theory, developed by Lorentz and J. J. Thomson, agree completely with those of the thermodynamic theory, while there is a disagreement between the results of Drude and Lorentz, which is not to be ascribed only to the different suppositions of the calculations, Drude assuming two or more different kinds of free corpuscles, while Lorentz and J. J. Thomson admit only one kind of free corpuscles, in agreement with the fact, that we know only one kind of these minute particles. The agreement of the results derived from two quite different theories is the more remarkable as it is not what we should expect. Indeed, according to the corpuscular theory, the thermoelectric phenomena are very closely connected with the conduction of heat, taking place in the circuit, while in the thermodynamic theory, we are obliged to leave aside this conduction, as an irreversible process. Therefore the question arises, how the above-stated agreement between the results of the two theories is possible. An attempt will be made to answer this question in the following paper.

According to the corpuscular theory of electric conduction, developed by Riecke, Drude, and Lorentz, the currents of electricity and heat are carried by negatively charged corpuscles, diffused as a very light gas throughout the metal, having a mean free path and a mean velocitv, and exerting a definite pressure. J. J. Thomson has lately shown that the theory in its usual form requires the presence of so many corpuscles that the specific heat would exceed by far the actual specific heat of the metal. J. J. Thomson has in addition proposed a modification of the theory which is not

* Riecke, Annalen der Physik, lxvi. pp. 353, 545, 1199 (1898).

+ Drude, Annalen der Physik, i. p. 566 (1900); iii. p. 369 (1900).

$\ddagger$ Lorentz, 'Proceedings' Acad. of Amsterdam, pp. 438, 585, 684 (1904-05). $(1907)$. 
open to this objection, which makes the ratio of the conductivities for heat and electricity of the right magnitude, and gives an account of the radiation of heat and of the different effects called after Hall, Peltier, and Thomson. I shall, however, begin with the first method, supposing the corpuscles to be free and in temperature equilibrium with their surroundings like a very rarefied gas in a porous body. The following considerations apply to the first as well as to the second theory.

\section{$\S 1$. Potential-Differences between Metals in Contact.}

When two metals $A$ and $B$ at the same temperature are placed in perfect contact, if the number $N_{1}$ of corpuscles in unit volume in the metal $A$ is greater than that in $B$, then corpuscles will flow from $A$ to $B$. There is evaporation of negative electricity in the first metal and condensation in the second. After a very short time, however, this distillation will be stopped, the metal A getting charged positively and the metal $B$ negatively. These charges produce an electric force $X$ directed from $A$ to $B$. The attraction of the positive electricity in $A$ will tend to prevent the corpuscles escaping from it, and the flow will cease, when the attraction of the positive electricity in $A$ and the repulsion of the negative in $B$ just balances the effect of the difference in pressure. The positive electrification in $A$ and the negative in $B$ will be close to the surface of separation, and these two electrifications will produce a difference in electric potential between $A$ and $B$, which we can calculate in the following way.

Let $\mathrm{N}$ be the number of corpuscles per unit volume in $\mathrm{A}$ at a point distant $x$ from the boundary between the two metals. Then the number of corpuscles crossing unit surface at $x$ in unit time according to the law of diffusion is equal to ? $v l . \partial N / \partial x$, where $v$ denotes the mean velocity and $l$ the mean free path of the corpuscles at the temperature $T$. Let $e$ be the negative charge of a corpuscle and $\mathrm{X}$ the electric force at the point $x$, then the number of corpuscles acted on by the force $\mathrm{X}$, passing through unit surface in unit time, is equal to NelvX/4aT, $\alpha$ being the universal constant of the kinetic theory of gases. The number of corpuscles, flowingin unit time through unit surface from $A$ to $B$, is equal to

$$
\frac{1}{3} v l \frac{\partial \mathrm{N}}{\partial x}-\frac{\mathrm{N} e l v \mathrm{X}}{4 \alpha \mathrm{I}}
$$

in the case of equilibrium this flux must vanish. Therefore

$$
\mathrm{X}=\frac{4}{3} \frac{\boldsymbol{\alpha}}{e} \mathrm{~T} \frac{\partial \mathrm{N}}{\partial x} \frac{1}{\mathrm{~N}} \text {. }
$$

Phil. Mag. S. 6. Vol. 16. No. 95. Nov, 1908. 
Let us suppose that there is a thin layer between the substances $\mathrm{A}, \mathrm{B}$ in which the transition from $\mathrm{A}$ to $\mathrm{B}$ takes place gradually, and let us reckon the abscissa $x$ from one of the boundaries of this layer.

$$
\begin{gathered}
\mathrm{X} d x=\frac{4}{3} \frac{\alpha}{e} \mathrm{~T} \frac{\partial \log \mathrm{N}}{\partial x} d x \\
\int_{\mathbf{A}}^{\mathrm{B}} \mathrm{X} d x=\mathrm{V}_{a}-\mathrm{V}_{b}=\frac{4}{3} \frac{\alpha}{e} \mathrm{~T} \log \frac{\mathrm{N}_{2}}{\mathrm{~N}_{\mathrm{I}}} .
\end{gathered}
$$

$V_{a}$ and $V_{b}$ denote the potentials in the metals $A$ and $B$, $N_{1}$ the number of free corpuscles in the metal $A, N_{2}$ that in metal B.

The potential-differences which arise in this way are not comparable with the Volta differences of potential between metals in contact. The experimental results, however, cannot be in accord with this theoretical calculation for well known reasons. Let us remark, in addition, that we have assumed a perfect metallic contact; if this condition is not satisfied quite different phenomena take place, when an electric force is acting between two metals or even between two pieces of the same metal.

These new phenomena occur in the coherer, which we shall consider later on in the light of the second theory of metallic conduction due to J. J. Thomson.

\section{\$2. Thermoelectric Force in an unequally heated Metallic Bar.}

Let $A B$ be a bar of metal, and let the temperature increase from $A$ to $B$. If we assume the number of corpuscles to be independent of the temperature, there is a diffusion of corpuscles with a large amount of kinetic energy from the hotter parts of the metal to the colder ones. In consequence of the collisions which the corpuscles make with the atoms of the metal, resulting in alterations in the energy, the corpuscles will carry heat from the hot to the cold parts of the metal. This transference of heat takes place without a change in the number of corpuscles in unit volume, without a transference of the electric charges, and therefore without the production of an electromotive force. This process is a completely irreversible one, like the diffusion of two volumes of a gas of different temperatures but of the same number of molecules per unit volume. No mechanical work can be produced by such a process. If the number of corpuscles per unit volume in a metal does not depend on the temperature, there can be no electromotive force along the bar to 
keep the corpuscles from drifting under the pressure differences. Let us now suppose that the number of corpuscles per unit volume depends on the temperature, so that at $100^{\circ} \mathrm{C}$. one c.cm. contains a larger number of these particles than one e.cm, at $0^{\circ} \mathrm{C}$.

Let the temperature increase from $A$ to $B$. If we consider in a distance $x$ from $A$ a cross-section of unit surface, a larger number of corpuscles will drift in unit time from

Fig. 1.

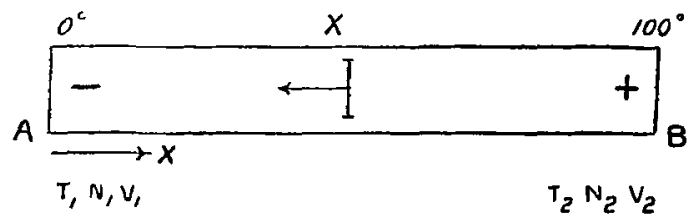

right to left than in- the opposite direction. An accumulation of negative charges will take place in $A$ at the colder end of the bar and of positive charges at the hotter end $B$. These charges produce a certain electromotive force $X$ at a point distant $x$ from $A$, tending to move the corpuscles from $A$ to $B$. The attraction of the positive electricity in B will tend to prevent the corpuscles escaping from it, and the flow will cease when the attraction of the positive electricity in B and the repulsion of the negative in $A$ just balance the effect of the difference in the number of corpuscles per unit volume due to the difference of the temperature. The same considerations as in the first paragraph, applied to this case, lead to the expression of the electric force $\mathrm{X}$

$$
\mathrm{X}=\frac{4}{3} \underset{e}{\frac{\alpha}{e}} \mathrm{~T} \frac{\partial \log \mathrm{N}}{\partial x} \text {. }
$$

$\mathrm{N}$ is the number of corpuseles in unit volume at $x$, and $\mathrm{T}$ the corresponding temperature, thus

$$
\mathrm{V}_{1}-\mathrm{V}_{2}=\frac{4}{3} \frac{\alpha}{e} \int_{\mathrm{N}_{1}}^{\mathrm{N}_{2}} \mathrm{~T} \frac{\partial \log \mathrm{N}}{\partial x} d x
$$

A potential-difference of this amount will be found between the two ends of the metallic bar. The electric force $X$ will account for the Thomson effect and for one part of the electric forces of thermoelectric couples. These forces $X$ may be used for the performance of mechanical work, which again could be transformed into heat, so that the process under consideration is a reversible one. 
\$3. The Electromotive Force of Thermo-elements.

Let us combine two metals $a$ and $b$ in a way indicated by fig. 2. By this arrangement four different differences of electric potential will occur, according to the two contacts

Fig. 2.

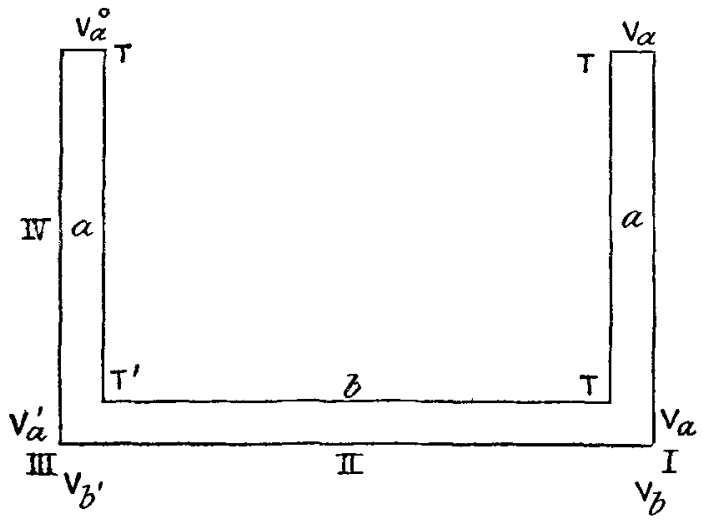

I. and III. at the temperatures $\mathrm{T}$ and $\mathrm{T}^{\prime}$, and according to the differences of temperature in the two metals $a$ and $b$.

$$
\begin{aligned}
\text { I. ... } \mathrm{V}_{a b}-\mathrm{V}_{b} & =\frac{4}{3} \frac{\alpha}{e} \mathrm{~T} \log \frac{\mathrm{N}_{b}}{\mathrm{~N}_{a},} \\
\text { II. ... } \mathrm{V}_{b}-\mathrm{V}_{b^{\prime}} & =\frac{4}{3} \frac{\alpha}{e} \int_{\mathrm{T}}^{\mathrm{T}^{\prime}} \mathrm{T} \frac{\partial \log \mathrm{N}_{b}}{\partial \mathrm{T}} d \mathrm{~T}, \\
\text { III. ... } \mathrm{V}_{b^{\prime}}-\mathrm{V}_{a^{\prime}} & =\frac{4}{3} \frac{\alpha}{e} \mathrm{~T}^{\prime} \log \frac{\mathrm{N}_{a^{\prime}}}{\mathrm{N}_{b^{\prime}}}, \\
\text { IV. ... } \mathrm{V}_{a^{\prime}}-\mathrm{V}_{a_{0}} & =\frac{4}{3} \frac{\alpha}{e} \int_{\mathrm{T}^{\prime}}^{\mathrm{T}} \mathrm{T} \frac{\partial \log \mathrm{N}_{a}}{\partial \mathrm{T}} d \mathrm{~T} .
\end{aligned}
$$

The thermoelectromotive force $\mathrm{E}=\mathrm{V}_{a}-\mathrm{V}_{a_{0}}$ is therefore

$$
\begin{aligned}
\mathrm{E}= & \frac{4}{3} \frac{\alpha}{e}\left\{\mathrm{~T}^{\prime} \log \frac{\mathrm{N}_{a^{\prime}}}{\mathrm{N}_{b^{\prime}}}-\mathrm{T} \log \frac{\mathrm{N}_{a}}{\mathrm{~N}_{b}}\right\} \\
& -\frac{4}{3} \frac{\alpha}{e}\left[\mathrm{~T} \log \frac{\mathrm{N}_{a}}{\mathrm{~N}_{b}}\right]_{\mathrm{T}}^{\mathrm{T}^{\prime}}-\int_{\mathrm{T}}^{\mathrm{T}^{\prime}} \log \frac{\mathrm{N}_{b}}{\mathrm{~N}_{a}} d \mathrm{~T} \cdot \frac{4}{3} \frac{\alpha}{e}, \\
\mathrm{E}= & \frac{4}{3} \frac{\alpha}{e} \int_{T}^{\mathrm{T}^{\prime}} \log \frac{\mathrm{N}_{a}}{\mathrm{~N}_{b}} d \mathrm{~T} .
\end{aligned}
$$


Suppose $\mathrm{N}_{a} / \mathrm{N}_{b}$ to be independent of the temperature, then

$$
\begin{aligned}
\mathrm{E} & =\frac{4}{3} \frac{\alpha}{e} \log \frac{\mathrm{N}_{a}}{\mathrm{~N}_{b}}\left(\mathrm{~T}^{\prime}-\mathrm{T}\right), \\
\mathrm{E} e & =\frac{4}{3} \log \frac{\mathrm{N}_{a}}{\mathrm{~N}_{b}}\left[\alpha^{\prime} \mathrm{T}^{\prime}-\alpha \mathrm{T}\right],
\end{aligned}
$$

the thermoelectromotive force would be proportional to the difference of temperature, and would only depend on the potential-differences of contact at the temperatures $\mathrm{T}^{\prime}$ and $\mathrm{T}$. If one single corpuscle passes round about the thermoelectric circuit, the force $\mathrm{E}$ will perform an amount of electric work equal to E.e. Neglecting a certain factor this work is equal to the difference of the values which the quantity $\alpha \mathrm{T}$ possesses at the temperatures $\mathrm{T}$ and $\mathrm{T}$. The difference $\alpha \mathrm{T}^{\prime}-\alpha \mathrm{T}$ is the increase in the mean kinetic energy of a molecule of gas, which is heated from the temperature $T$ to the temperature $\mathrm{T}^{\prime}$.

If we study the electromotive force produced by a standard metal, for instance lead combined with any other metal $a$, we may represent the electromotive force as a function of the temperature difference in the following way:

$$
\mathrm{E}=\alpha_{t} t+\frac{\beta_{a}}{2} t^{2},
$$

$\alpha_{c}$ and $\beta_{a}$ are the temperature coefficients of the metal $\alpha$; combining the standard metal with any other metal $b$ we find :

$$
\mathrm{E}=\alpha_{b} t+\frac{\beta_{b}}{2} t^{2},
$$

$\alpha_{b}$ and $\beta_{b}$ are the first and second coefficients of temperature of the metal $b$. If two metals $a$ and $b$ form a thermoelement, one junction of which is at the temperature $t$, the other at $0^{\circ} \mathrm{C}$., the thermoelectric force will be found to be:

$$
\begin{aligned}
& \mathrm{E}=\left(\alpha_{a}-\alpha_{b}\right) t+\left(\frac{\beta_{a}-\beta_{b}}{2}\right) t^{2}, \text { or } \\
& \mathrm{E}=\left(\alpha_{b}-\alpha_{b}\right)(\mathrm{T}-273)+\left(\frac{\beta_{a}-\beta_{b}}{2}\right)(\mathrm{T}-273)^{2},
\end{aligned}
$$

$\mathrm{T}$ being the absolute temperature.

$$
\begin{gathered}
\frac{d \mathrm{E}}{d \mathrm{~T}}=\frac{4}{3} \frac{\alpha}{e} \log \frac{\mathrm{N}_{a}}{\overline{\mathrm{N}}_{b}}=\alpha_{a}-\alpha_{b}+\left(\beta_{a}-\beta_{b}\right)(\mathrm{T}-273) \\
\overline{\mathrm{N}_{b}}=\epsilon^{\frac{3}{4} \frac{e}{a}\left[a_{a}-\alpha_{b}+\left(\beta_{a}-\beta_{b}\right)(\mathrm{T}-273)\right]} .
\end{gathered}
$$


This formula is symmetrical with regard to the coefficients of the metals $a$ and $b$. The two metals playing the same part, we may conclude from this symmetry that the numbers of free corpuscles in the two metals are the following functions of the temperature:

$$
\begin{aligned}
& \mathrm{N}_{a}=\mathrm{C}_{\epsilon} \frac{3 \frac{e}{4}}{a^{2}\left[a_{a}+\beta_{a}(\mathrm{~T}-273)\right]}, \\
& \mathrm{N}_{b}=\mathrm{C}_{\epsilon} \frac{3}{4}^{\frac{e}{\alpha}}\left[\alpha_{b}+\beta_{b}(\mathrm{~T}-273)\right] .
\end{aligned}
$$

The last step is of course not conclusive. For suppose

and

$$
\begin{aligned}
& \mathrm{N}_{a}=\mathrm{C}_{\epsilon^{\frac{3}{4}} \frac{e}{a}\left[\alpha_{a}+\beta a(\mathrm{~T}-273)+\gamma \mathrm{T}^{2}\right],} \\
& \mathrm{N}_{b}=\mathrm{C}_{\epsilon^{\frac{3}{4}} \frac{e}{a}\left[a b+\beta b(\mathrm{~T}-273)+\gamma \mathrm{T}^{2}\right],}
\end{aligned}
$$

we should find again

$$
\begin{aligned}
& \mathrm{N}_{a}=e^{\frac{3}{4} \frac{e}{a}\left[\alpha_{a}-a b+\left(\beta_{a}-\beta b\right)(\mathrm{T}-273)\right]}, \\
& \mathrm{N}_{b}=\mathrm{C}_{1}+\mathrm{C}_{1} \frac{3}{4} \frac{e}{\alpha} \beta_{a} \mathrm{~T}+\ldots \ldots . .
\end{aligned}
$$

The number of free corpuscles will vary approximately as the absolute temperature. $\beta_{a}$ for some metals and for certain intervals of temperature of other metals being negative, we see that in these cases the number $\mathrm{N}_{a}$ will decrease with increasing temperature. The electric conductivity is proportional to $\frac{\mathrm{N} l v}{\mathrm{~T}}$, and by experiment it has been shown to be proportional to $\mathrm{T}^{-1}$; thus, $\mathrm{N}$ being approximately proportional to $\mathrm{T}$, and $v$ proportional to $\mathrm{T}^{1 / 2}, l$ the mean free path of the corpuscles must vary approximately as $T^{-3 / 2}$. The variation of the number of free corpuscles with the temperature involves a still more rapid variation of the mean free path. Thus the effects which depend on the free path, such as the effect of magnetic force on electrical resistance or the absorption of light by the metal, would be greatly influenced by the lowering of the temperature.

\section{\$4. The Peltier Effect.}

Returning to the suppositions of $\S 1$, we find an electric force acting at the junction of two metals, produced by the different number of corpuscles per unit volume in the two 
substances. The potential-difference arising in this way is equal to

$$
\mathrm{V}_{a}-\mathrm{V}_{b}=\frac{4}{3} \frac{\alpha}{e}-\mathrm{T} \log \frac{\mathrm{N}_{2}}{\mathrm{~N}_{1}}
$$

If a current $i$ is flowing through the metals in a direction opposite to the electric force

$$
\mathrm{X}=\frac{4}{3} \frac{x}{e} \frac{\partial \log \mathrm{N}}{\partial x},
$$

a corpuscle in travelling from $B$ to $A$, and acted on by the foree $\mathrm{X} e$, will increase its kinetic energy, and therefore abstraet from the metal an amount of heat whose mechanical equivalent is $\mathrm{X} e d x$. If the current $i$ is flowing in the direction opposite to $X$, the number of corpuscles which cross unit area in unit time is $\frac{i}{e}$, and the mechanical equivalent of the heat they abstract from the metals passing through the junction is

$$
\mathrm{Q}^{\prime}=\frac{i}{e} \cdot \int_{\mathrm{B}}^{\mathrm{A}} \mathrm{X} e d x=i\left(\mathrm{~V}_{b}-\mathrm{V}_{a}\right)=i \cdot \frac{\alpha}{e} \frac{4}{3} \mathrm{~T} \log \frac{\mathrm{N}_{1}}{\mathrm{~N}_{2}}
$$

The heat absorbed in unit time and per unit curcent, i.e. the Peltier effect, will be :

$$
\mathrm{Q}=\frac{4}{3} \frac{\alpha}{e} \mathrm{~T} \log \frac{\mathrm{N}_{1}}{\mathrm{~N}_{2}}=\mathrm{V}_{\iota}-\mathrm{V}_{a}
$$

If the current were to flow in the opposite direction the same amount of heat would be communicated to the metal. The Peltier effect is a real measure of the potential-differences between metals in contact. From the Peltier effect we may derive the ratio of the number of corpuscles in two metals. Thus to take the case of antimony and bismuth, whose Peltier effect is exceptionally large,

$$
\log \frac{N_{1}}{N_{2}}=1 \cdot 33 \quad \text { or } \quad \frac{N_{1}}{N_{2}}=3 \cdot 8 \text {. }
$$

Thus, if the number of corpuscles in the unit volume of antimony were about four times that in bismuth we should, on this theory, get Peltier effects of about the right amount. The Peltier effect for antimony and bismuth being very much larger than that for most pairs of metals, we see that the theory indicates that the number of free corpuseles per unit volume does not vary much from one metal to another. 
Now Professor J. J. Thomson*, by a comparison of the numbers of free corpuscles and the mean free path in different metals, came to the conclusion that the mechanism by which, according to this first corpuseular theory, the electric current is supposed to be conveyed, is at most only a part and not the whole of the process of metallic conduction.

\section{\$5. The Thomson Effect.}

If the number of corpuscles in unit volume of a metal increases with increasing temperature, there must be electric

Fig. 3.

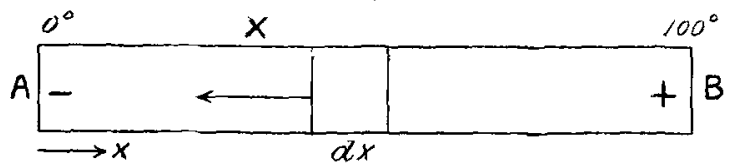

forces $\mathrm{X}$ along the bar, which, following the notation of $\S 2$, are found to be equal to

$$
\mathrm{X}=\frac{4}{3} \frac{\alpha}{e} \mathrm{~T} \frac{\partial \log \mathrm{N}}{\partial x}
$$

and tend to move the corpuscles from left to right. Hence a corpuscle in travelling from $x+d x$ to $x$, will abstract from the metal an amount of heat whose mechanical equivalent is $\mathrm{X} e d x$, or

$$
\frac{4}{3} \alpha \mathrm{T} \frac{\partial \log \mathrm{N}}{\partial x} d x=\frac{4}{3} \alpha \mathrm{T} \frac{\partial \log \mathrm{N}}{\partial \mathrm{T}} d \mathrm{~T}
$$

The corpuscle when at $x+d x$ has an amount of kinetic energy equal to $\alpha\left(\mathrm{T}+\frac{\partial \mathrm{T}}{\partial x} d x\right)$, while at $x$ its kinetic energy is equal to $\alpha \mathrm{T}$; hence between $x+d x$ and $x$ the corpuscle will lose a part of its kinetic energy equal to $\alpha \frac{\partial T}{\partial x} d x$, which is communicated to the metal in the form of heat. The transference of this amount of heat is an irreversible process. Thus the total amount of heat communicated by the corpusele to the metal is :

$$
\begin{aligned}
& \alpha \frac{\partial \mathrm{T}}{\partial x} d x-\frac{4}{3} \alpha^{\prime} \mathrm{T} \frac{\partial \log \mathrm{N}}{\partial \mathrm{T}} d \mathrm{~T}, \quad \text { or } \\
& x\left(1-{ }_{3}^{4} \mathrm{~T} \frac{\partial \log \mathrm{N}}{\partial \mathrm{T}}\right) d \mathrm{~T} .
\end{aligned}
$$

* J. J. Thomson, ‘ Corpuscular Theory of Matter,' p. 75 (1907). 
If the current $i$ is flowing in the direction from $\mathrm{A}$ to $\mathrm{B}, i . e$. in the direction of increasing temperature, the number of corpuscles which cross unit area in unit time in the direction from $\mathrm{B}$ to $\mathrm{A}$ is $i / e$, and the mechanical equivalent of the heat they communicate to the metal between the places where the temperatures are respectively $\mathrm{T}$ and $\mathrm{T}+d \mathrm{~T}$ is equal to

$$
i \frac{\alpha}{e}\left(1-\frac{4}{3} \mathrm{~T} \frac{\partial \log \mathrm{N}}{\partial \mathrm{T}}\right) d \mathrm{~T} .
$$

But if $\sigma$ is the specific heat of electricity in the metal, this amount of heat is by definition equal to

$$
-i \sigma d \mathrm{~T} \text {, }
$$

the sign minus being applied because the electric current is flowing from the cold to the hot part of the metal; hence

$$
\sigma=-\frac{\alpha}{e}\left(1-\frac{4}{3} \mathrm{~T} \frac{\partial \log \mathrm{N}}{\partial \mathrm{T}}\right)
$$

As lead has no Thomson effect, its $\sigma$ is zero. If the current is flowing in the direction of decreasing temperature, and if in this case heat is developed, we consider $\sigma$ as positive ; thus $\sigma$ would be positive in the metals $\mathrm{Cu}$ commercial, $\mathrm{Sb}$ commercial, $\mathrm{Cd}, \mathrm{Zn}, \mathrm{Ag}$. On the other hand, $\sigma$ is negative in the metals : $\mathrm{Fe}, \mathrm{Hg}, \mathrm{Sn}, \mathrm{Al}, \mathrm{Pt}, \mathrm{Sb}$ pure, $\mathrm{Bi}$ pure.

In these metals heat will be absorbed when an electric current flows from hot to cold parts. When the specific heat $\sigma$ in the lead disappears we see that the corpuscle passing from $\mathrm{T}+d \mathrm{~T}$ to ' $\mathrm{T}$ loses the same amount of kinetic energy in consequence of the decreasing temperature as it gains in consequence of the acceleration produced by the pressure difference due to the increasing number of corpuscles with increasing temperature. Consider two metals $a$ and $b$, containing at the same temperature $T$ the numbers $\mathrm{N}_{a}$ and $\mathrm{N}_{b}$ of free corpuscles, the corresponding specific heat of electricity will be

$$
\begin{aligned}
\sigma_{a} & =\frac{4}{3} \frac{\alpha}{e} \mathrm{~T} \frac{\partial \log \mathrm{N}_{a}}{\partial \mathrm{T}}-\frac{\alpha}{e}, \\
\sigma_{b} & =\frac{4}{3} \frac{\alpha_{\mathrm{T}}}{e} \frac{\partial \log \mathrm{N}_{b}}{\partial \mathrm{T}}-\frac{\alpha}{e}, \\
\sigma_{a}-\sigma_{b} & =\frac{4}{3} \frac{\alpha}{e} \mathrm{~T} \frac{\partial \log \frac{\mathrm{N}_{a}}{\mathrm{~N}_{b}}}{\partial \mathrm{T}} .
\end{aligned}
$$


\$6. Connexion between the different Effects.

The results hitherto arrived at are as follows :-

If the number of free corpuscles increases with increasing temperature there is an electric force along an unequally heated metallic bar, given by the expression

$$
\mathrm{X}=\frac{4 \alpha}{3 e} \mathrm{~T} \frac{\partial \log \mathrm{N}}{\partial \mathrm{T}} \frac{\partial \mathrm{T}}{\partial r} \text {. . . . . . }
$$

The electromotive force of a thermoelement is equal to

$$
\mathrm{E}=\frac{4}{3} \frac{a}{e} \int_{\mathrm{T}}^{\mathrm{T}^{\prime}} \log \frac{\mathrm{N}_{a}}{\mathrm{~N}_{b}} d \mathrm{~T} . . . . . .
$$

The Peltier effect between two metals at the tomperature $T$ is equal to

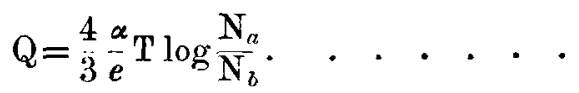

The Thomson effect of a metal is equal to:

$$
\begin{aligned}
& \sigma=\frac{4}{3} \frac{\alpha}{e} \mathrm{~T} \frac{\partial \log \mathrm{N}_{a}}{\partial \mathrm{T}^{\prime}}-\frac{\alpha}{e} . . . .
\end{aligned}
$$

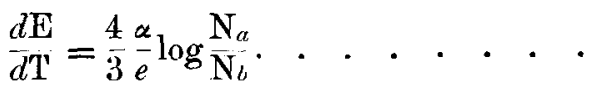

$$
\begin{aligned}
& \frac{d^{2} \mathrm{E}}{d \mathrm{~T}^{2}}=\frac{4}{3} \frac{a^{\partial \log \frac{\mathrm{N}_{a}}{\mathrm{~N}_{L}}}}{\partial \mathrm{T}} \text {. . . . . . . . }
\end{aligned}
$$

Comparing the equations 3 and $2^{\prime}$ we get

$$
\begin{aligned}
& \mathrm{Q}=\mathrm{T} \frac{d \mathrm{E}}{d \mathrm{~T}^{\top}} \cdot \quad \cdot \quad \cdot \quad \cdot \cdot \cdot \cdot \cdot \cdot
\end{aligned}
$$

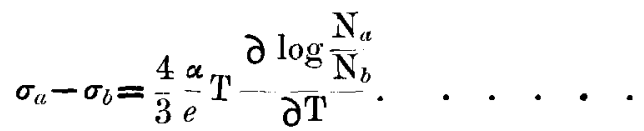

Comparing the equations $4^{\prime}$ and $2^{\prime \prime}$ we get

$$
\sigma_{a}-\overline{\sigma_{b}}=\mathrm{T} \frac{d^{2} \mathrm{E}}{d \mathrm{~T}^{2}}=\mathrm{T} \frac{d}{d^{\prime} \mathrm{\Gamma}}\left(\frac{\mathrm{Q}}{\mathrm{T}}\right) . \quad . \quad . \quad . \quad .
$$

Lord Kelvin has from thermodynamic principles derived the equations 5 and 6 . The results of the thermodynamic theory agree with those of the corpuscular theory of metals. We see immediately why we are allowed in the thermodynamic theory to neglect the irreversible process of thermal 
conduction. In the corpuscular theory of the laws of thermoelectricity too we do not consider the predominant part of the heat conduction, but take into account only the small reversible part of the conduction, which depends on the variation in the number of free corpuscles in unit volume. This variation gives rise to an electric force along an unequally heated bar of metal. All the phenomena connected with this force are reversible. According to this theory, a current of heat is to a small extent connected with a reversible process, able to perform mechanical work. This is, I think, the reason for the agreement of the two theories, whose assumptions are quite different. The corpuscular theory, however, affords a deeper insight into the nature of the physical processes, and gives expressions for the electric force $\mathrm{X}$ and for the electromotive force $\mathrm{E}$, depending on the number of free corpuscles. According to the experimental law:

$$
\begin{gathered}
\frac{d \mathrm{E}}{d \mathrm{~T}}=\alpha_{a}-\alpha_{b}+\left(\beta_{a}-\beta_{b}\right)(\mathrm{T}-273), \\
\mathrm{Q}=\mathrm{T} \frac{d \mathrm{E}}{d \mathrm{~T}}=\left(\alpha_{a}-\alpha_{b}\right) \mathrm{T}+\left(\beta_{a}-\beta_{b}\right)(\mathrm{T}-273) \mathrm{T} .
\end{gathered}
$$

If we keep the temperature of a junction between two different metals $a$ and $b$ constant at $0^{\circ} \mathrm{C}$. the Peltier effect will be

$$
\mathrm{Q}_{0}=\left(\alpha_{a}-\alpha_{b}\right) 273 \text {. }
$$

It a current of 1 ampere is flowing from copper to the other metal in the following table, the beat developed or

\begin{tabular}{|c|c|c|c|}
\hline & $\begin{array}{l}\text { Calculated } \\
\text { from numbers } \\
\text { of Riecke. }\end{array}$ & $\begin{array}{l}\text { Calculated } \\
\text { from numbers } \\
\text { of Olemencic. }\end{array}$ & $\begin{array}{l}\text { Observed } \\
\text { by Jabn *. }\end{array}$ \\
\hline $\mathrm{Cu}-\mathrm{Pt} \ldots \ldots$ & $+0 \cdot 292$ & +0.327 & +0.320 \\
\hline $\mathrm{Cu}-\mathrm{Ag} \ldots \ldots$ & -0.183 & -0.495 & -0.413 \\
\hline $\mathrm{Cu}-\mathrm{Fe} \ldots \ldots$ & $-3 \cdot 762$ & $-2 \cdot 64$ & $-3 \cdot 163$ \\
\hline $\mathrm{Cu}-\mathrm{Zn} \ldots \ldots$ & $-0 \cdot 207$ & -0.353 & -0585 \\
\hline $\mathrm{Cu}-\mathrm{Cd} \ldots \ldots$ & --0.306 & -0.617 & $-0 \cdot 616$ \\
\hline $\mathrm{Cu}-\mathrm{Ni} \ldots \ldots$ & +4.37 & $+4 \cdot 68$ & $+4 \cdot 362$ \\
\hline
\end{tabular}
absorbed in an hour, measured in calories, will bave the values indicated as follows:-

* Jahn, Wiedemann's Annalen, Bd. xxxiv. p. 763 (1888). 


$$
\sigma_{a}-\sigma_{b}=\mathrm{T} \frac{d^{2} \mathrm{E}}{d \mathrm{~T}^{2}}
$$

but

hence,

$$
\frac{d^{2} \mathrm{E}}{d \mathbf{T}^{2}}=\beta_{a}-\beta_{b},
$$

therefore

$$
\sigma_{a}-\sigma_{b}=\mathrm{T}\left(\beta_{a}-\beta_{b}\right)
$$

$$
\sigma_{a}=\mathrm{T} \cdot \beta_{a}-\frac{\alpha}{e}, \quad \sigma_{b}=\mathrm{T} \cdot \beta_{b}-\frac{\alpha}{e} \text {. }
$$

Admitting the number of free corpuscles to be the simple function of the temperature as indicated by the formula

$$
\mathrm{N}_{a}=\mathrm{C}_{\epsilon} \frac{3 \frac{e}{4}}{a}\left[a_{a}+\beta_{a(1}(\mathrm{T}-273)\right],
$$

we get

$$
\begin{aligned}
\log \mathrm{N}_{a} & =\mathrm{C}+\frac{3}{4} \frac{e}{\alpha}\left(\alpha_{a}+\beta_{a} \mathrm{~T}\right) \\
\frac{\partial \log \mathrm{N}_{a}}{\partial \mathrm{T}} & =\frac{3}{4} \frac{e}{\alpha} \beta_{a},
\end{aligned}
$$

but

$$
\sigma_{a}=\frac{4}{3} \frac{a}{e} \mathrm{~T} \frac{\partial \log \mathrm{N}_{a}}{\partial \mathrm{T}}-\frac{\alpha^{-}}{e}
$$

hence

$$
\sigma_{a}=\mathrm{T} \beta_{a}-\frac{\alpha}{e} .
$$

This second deduction of the value of $\sigma_{a}$ is only possible if $\mathrm{N} a$ is of the form indicated above, and not of the form

$$
\mathrm{N}_{a}=\mathrm{C}^{\frac{3}{4} \frac{e}{a}\left[\alpha_{a}+\beta a(\mathrm{~T}-273)+\gamma^{\mathrm{T}} \mathrm{2}\right]} \text {. }
$$

If a current of 10 amperes is flowing through bismuth in the direction of decreasing temperature, the heat developed between two cross-sections, whose temperature differences are $1^{\circ} \mathrm{C}$., in unit time will be $\sigma=24 \cdot 5 \cdot 10^{-6} \mathrm{gr}$. cal.

For $\mathrm{Hg}$ the Thomson effect would be $-6 \cdot 9 \cdot 10^{-6} \mathrm{gr}$. cal.* Combining these results of absolute measurements with a series of relative observations, we shall find the observed values of the Thomson effect united in the following table, which contains moreover the values of the quantity $\mathrm{T} \beta_{a}$ and $\mathrm{T} \beta_{a}-\alpha / e . \quad \mathrm{T}=273, \mathrm{~A}=4 \cdot 2 \cdot 10^{7}$ mechanical equivalent of 1 calory, $e=1 \cdot 2 \cdot 10^{-20}, a=1 \cdot 5 \cdot 10^{-16}, \frac{\alpha}{e} \frac{1}{\mathrm{~A}}=0 \cdot 3 \cdot 10^{-3}$.

* Hagen, Wied. Ann. Bd. xxviii. p. 199 (1886). 
Theory of the Thermoelectric Forces.

\begin{tabular}{|c|c|c|c|}
\hline & $\begin{array}{c}\pi_{a l} \cdot 10^{6} \\
\text { observed. }\end{array}$ & $\mathrm{T} \beta_{l l} .10^{c}$. & $\left(\mathcal{L}_{\beta a}-\frac{a}{e \mathrm{~A}}\right) \cdot 10^{\prime}$. \\
\hline 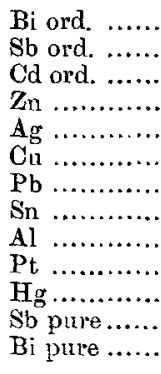 & $\begin{array}{r}+24.5 \\
+21.6 \\
+10.2 \\
+30 \\
+18 \\
+06 \\
0 \\
-0.04 \\
-0.04 \\
-60 \\
-69 \\
-7.8 \\
-102\end{array}$ & $\begin{array}{l}+28 \cdot 1 \\
+15 \cdot 68 \\
+9 \cdot 8 \\
+6 \cdot 21 \\
0 \\
+359 \\
+255 \\
-60\end{array}$ & $\begin{array}{l}-272 \\
-281 \\
-290 \\
-294 \\
-296 \\
-297 \\
-306\end{array}$ \\
\hline
\end{tabular}

Only the values of commercial bismuth and of mercury correspond to absolute measurements, and for these two metals the second coefficient $\beta_{a}$ of the thermoelectric force is unknown. A larger nunber of absolute determinations of the Thomson effeet in the same time as the exact measurements of $\beta_{a}$ are very necessary. The value $\mathrm{T} \beta_{a}$ is not much greater than the specific heat of electricity, being at any rate of the same order of magnitude as $\sigma_{\alpha}$. The theoretical value $\mathrm{T} \beta_{a}-\frac{\alpha}{e \mathrm{~A}}$, however, is not-even of the same order of magnitude as the observed value of the specific heat of electricity. There is a serious disagreement between the theory and the experimental result.

We assumed in paragraph 5 that a corpuscle, moving from $x+d x$ to $x$ or from $\mathrm{T}+d \mathrm{~T}$ to $\mathrm{T}$, will lose a part of its kinetic energy equal to $\alpha d \mathrm{~T}$ which is communicated to the metal in the form of heat. This transference of heat would take place if the corpuscles were drifting, but under the action of the difference of temperature. The heat motion of the corpuscles not being much affected by electric forces which we are able to apply on conductors, the heat communicated by the corpuscles to the metal in the interval of temperature $d^{\prime} \mathrm{T}$ as consequence of the electric force, is not ad' $\mathrm{T}$, but $a d \mathrm{~T} . \mathrm{C}$, where $C$ denotes a certain constant, smaller than unity. Thus we find $\sigma$ to be equal to

$$
\sigma=-\frac{\alpha}{e}\left(\mathrm{C}-\frac{4}{3} \mathrm{~T} \frac{\partial \log \mathrm{N}}{\partial \mathrm{T}}\right)
$$

From the numbers of the last table we derived the following 
values of $\mathrm{C}$, which, however, are not to be considered as definite figures.

$\begin{array}{lll}\mathrm{Cd} & \ldots \ldots \ldots \ldots & 0.0604 \\ \mathrm{Zn} & \ldots \ldots \ldots \ldots . & 0.0404 \\ \mathrm{Ag} & \ldots \ldots \ldots \ldots . & 0.0207 \\ \mathrm{Cu} & \ldots \ldots \ldots \ldots . & 0.0186 \\ \mathrm{Sn} & \ldots \ldots \ldots \ldots . & 0.0119\end{array}$

If $\mathrm{C}$ had really different values for various metals $\sigma_{a}-\sigma_{b}$ would be equal to

$$
\frac{4}{3} \frac{\alpha}{e}\left(\mathrm{~T}-\frac{\partial \log \frac{\mathrm{N}_{a}}{\mathrm{~N}_{b}}}{\partial \mathrm{T}}\right)-\frac{\alpha}{e}\left(\mathrm{C}_{a}-\mathrm{C}_{b}\right),
$$

and there would be no more agreement between the thermodynamical and corpuscular theory of the Thomson effect.

\$ 7. On the Electric Force $\mathrm{X}$ and Pyroelectricity.

We found

$$
\begin{aligned}
\sigma & =\frac{4}{3} \frac{\alpha}{e} \mathrm{~T} \frac{\partial \log \mathrm{N}_{a}}{\partial \mathrm{T}}-\frac{\alpha}{e}, \\
\sigma & =\frac{4}{3} \frac{\alpha}{e} \mathrm{~T} \frac{\partial \log \mathrm{N}_{a}}{\partial x} \frac{\partial x}{\partial \mathrm{T}}-\frac{\alpha}{e}, \\
\mathrm{X} & =\frac{4}{3} \frac{\alpha}{e} \mathrm{~T} \frac{\partial \log \mathrm{N}_{a}}{\partial x} ;
\end{aligned}
$$

hence

$$
\begin{aligned}
\sigma & =\mathrm{X} \frac{\partial x}{\partial \mathrm{T}}-\frac{\alpha}{e} \\
\mathrm{X} d x & =\left(\sigma+\frac{\alpha}{e}\right) d \mathrm{~T} \\
\int_{A}^{\mathrm{B}} \mathrm{X} d x & =\mathrm{V}_{1}-\mathrm{V}_{2}=\frac{\alpha}{e}\left(\mathrm{~T}_{2}-\mathrm{T}_{1}\right)+\int_{\mathrm{T}_{1}}^{\mathrm{T}_{2}} \sigma d \mathrm{~T} .
\end{aligned}
$$

But

$$
\sigma=\mathrm{T} \beta_{a}-\frac{\alpha}{e}
$$

hence

$$
\mathrm{V}_{1}-\mathrm{V}_{2}=\frac{\beta_{a}}{2}\left(\mathrm{~T}_{2}{ }^{2}-\mathrm{T}_{1}{ }^{2}\right)
$$

From this corpuscular theory of thermoelectricity we should expect a potential-difference between the ends of a metallic bar whose temperature varies from $T_{2}$ to $T_{1}$. This 
potential-difference has never been measured, but it ought to be measurable by means of an electrometric method. For iron $\beta_{a}$ is equal to $-4 \cdot 87$; if we put $\mathrm{T}_{2}=373^{\circ}, \mathrm{T}=273$ we find $V_{1}-V_{2}=4 \cdot 87 \cdot 6 \cdot 46 \cdot 10^{4} \cdot 10^{-8}=0 \cdot 0031$ volt. Since the actual value of the specific heat of electricity does not agree with the theoretical value, we cannot predict exactly the value of the electric force. If we suppose $\sigma_{a}$ to be independent of the temperature, and if, in order to get a better agreement between the actual and the theoretical value of $\sigma_{a}$, we neglect the term $\alpha / e$, we find for a bar of bismuth whose difference of temperature is $100^{\circ} \mathrm{C}$, a potential-difference of 0.00102 volt, in the case of iron 0.00030 volt.

Though the forces $\mathrm{X}$ have not yet been detected we might suspect them to exist not only in metals of different temperatures, but even in other bodies, whose temperature varies from point to point. Thus the phenomena known under the name of pyroelectricity may to a certain extent be ascribed to forces of the same origin as the forces $X$, a difference of temperature being connected with a difference of electric potential. The phenomena of pyroelectricity, occurring in crystals of every crystallographic system, depend, however, in a very complicated way on the structure of the crystal. It is interesting to remark, that even crystals of bismuth exhibit these phenomena. If electric poles in the crystals are to be found not only by differences of temperature, but also under the influence of pressure and of rays of light, these latter effects may be reduced to the former ones.

\section{\$. Conservation of Energy.}

Let us consider a thermoelectric circuit made up of two metals $a$ and $b$, whose junctions are at the constant tempera-

Fig. 4.

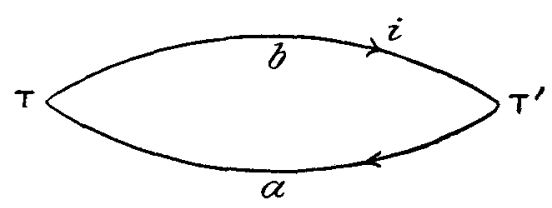

tures $\mathrm{T}$ and $\mathrm{T}^{\prime}$. Then the thermoelectromotive force $\mathrm{E}$ will give rise to an electric current $i$; and the amount of heat absorbed in unit time by the Peltier and Thornson effects 
will be :

$$
\begin{aligned}
\mathrm{W} & =i\left(\mathrm{Q}^{\prime}-\mathrm{Q}\right)+i \int_{\mathrm{T}}^{\mathrm{T}^{\prime}}\left(\sigma_{b}-\sigma_{a}\right) d \mathrm{~T} \\
& =i \frac{4}{3} \frac{\alpha}{e}\left\{\mathrm{~T}^{\prime} \log \left(\frac{\mathrm{N}_{a}}{\mathrm{~N}_{b}^{-}}\right)_{\mathrm{T}^{\prime}}-\mathrm{T} \log \left(\frac{\mathrm{N}_{a}}{\mathrm{~N}_{b}}\right)_{\mathrm{T}}\right\} \\
& +i \frac{4}{3} \frac{\alpha}{e} \int_{\mathrm{T}^{\prime}}^{\mathrm{T}} \mathrm{T} \frac{\partial \log \frac{\mathrm{N}_{a}}{\mathrm{~N}_{b}}}{\partial \mathrm{T}} d \mathrm{~T} \\
& =i \frac{4}{3} \frac{\alpha}{\frac{2}{\mathrm{~T}}}\left\{\mathrm{~T}^{\mathrm{V}} \log \left(\frac{\mathrm{N}_{a}}{\overline{\mathrm{N}_{b}}}\right)_{\mathrm{T}^{\prime}}-\mathrm{T} \log \left(\frac{\mathrm{N}_{a}}{\mathrm{~N}_{b}}\right)_{\mathrm{T}}\right\} \\
& +i \frac{4}{3} \frac{\alpha}{e}\left[\mathrm{~T} \log \frac{\mathrm{N}_{a}}{\mathrm{~N}_{b}}\right]_{\mathrm{T}^{\prime}}^{\mathrm{T}}+i \frac{4}{3} \frac{\alpha}{e} \int_{\mathrm{T}^{\prime}}^{\mathrm{T}^{\prime}} \log \frac{\mathrm{N}_{a}}{\mathrm{~N}_{b}} d \mathrm{~T},
\end{aligned}
$$

but

$$
\mathrm{E}=\frac{4}{3} \frac{\alpha}{e} \int_{\mathrm{T}}^{\mathrm{T}^{\prime}} \log \frac{\mathrm{N}_{a}}{\mathrm{~N}_{b}} d \mathrm{~T}
$$

bence $W=i \mathrm{E}$.

The work done by the current $i$ under the influence of the electromotive force $\mathrm{E}$ in unit time is equal to the heat absorbed in the circuit in the same time.

\section{§9. Resistance of Alloys.}

Lord Rayleigh * has pointed out that in the case of a mixture of metals there is, owing to their thermoelectric properties, something which cannot be distinguished by experiments from resistance, and which is absent when the metals are pure. Let us suppose that the mixed metals are arranged in thin layers, the adjacent layers being of different metals, and that the current passes through the body at right angles to the faces of the layer. When a current passes

Fig. 5.

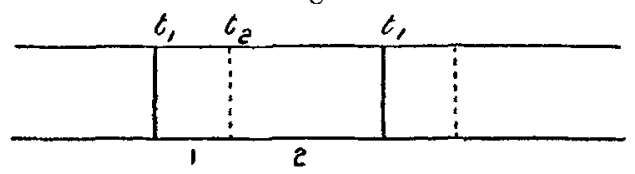

across the junction of two metals there is absorption or production of heat, proportional to the current passing across

* Lord Rayleigh, Collected Works, vol. iv. p. 232. 
the junction. Thus, when the current passes through the system of alternate layers of the two metals, one face of each layer will be cooled and the other heated, and thus in the pile of layers differences of temperature proportional to the current will be established. This will give rise to a thermoelectric force tending to oppose the current. Such a force would produce exactly the same effect as a resistance.

If $\Delta t$ is the difference of temperature between two junctions, the electromotive force of an elementary couple will be equal to

$$
e=\frac{4}{3} \frac{\alpha}{e} \log \frac{\mathrm{N}_{a}}{\mathrm{~N}_{b}} \Delta t
$$

The amount of heat developed in unit time and per unit area of the cross-section in each couple is equal to

$$
i Q_{1}+i \sigma_{1} \Delta t \text {. }
$$

This amount is to be equated to the heat conducted per second towards the cold junctions on the two sides. Let $k_{1}, k_{2}$ be the conductivities for heat of the two metals, $l_{1}$ and $l_{2}$ the corresponding lengths; the heat conducted per second towards the cold junctions is

$$
\stackrel{\Delta t}{l_{1}^{-}} k_{1}+\frac{\Delta t}{l_{2}} k_{2}=\Delta t\left(\frac{k_{1}}{l_{1}}+\frac{k_{2}}{l_{2}}\right) \frac{1}{\left(\frac{l_{1}+l_{2}}{l_{1}+l_{2}}\right)}=\Delta t \cdot n \cdot\left(\frac{k_{1}}{p_{1}}+\frac{k_{2}}{p_{2}}\right),
$$

if there are $n$ similar couples per unit of length,

and if we put

$$
n\left(l_{1}+l_{2}\right)=1
$$

$$
\frac{l_{1}}{l_{1}+l_{2}}=p_{1} \quad \text { and } \quad \frac{l_{2}}{l_{1}+l_{2}}=p_{2}
$$

when a stationary state is reached,

$$
i\left(\mathrm{Q}_{1} \pm \sigma_{1} \Delta t\right)=n \Delta t\left(\frac{k_{1}}{p_{1}}+\frac{k_{2}}{p_{2}}\right) \text {. }
$$

The whole electromotive force per unit of length is equal to

$$
n e=\omega^{\prime} i=\omega^{\prime} \frac{n \Delta t\left(\frac{k_{1}}{p_{1}}+\frac{k_{2}}{p_{2}}\right)}{Q_{1} \pm \sigma_{1} \Delta t},
$$

$\omega^{\prime}$ denoting the false resistance due to thermoelectric causes, Phil. Mag. S. 6. Vol. 16. No. 95. Nov. 1908. 3 F 
786 Dr. J. Kunz on the Corpuscular

$$
\begin{aligned}
\omega^{\prime} & =\frac{e\left(\mathrm{Q}_{1} \pm \sigma_{a} \Delta t\right)}{\Delta t\left(\frac{k_{1}}{p_{1}}+\frac{k_{2}}{p_{2}}\right)} \quad \mathrm{T}_{1}=t_{1}+273 \\
& =\frac{\frac{4}{3} \frac{\alpha}{e} \log \frac{\mathrm{N}_{a}}{\mathrm{~N}_{b}} \Delta t\left(\frac{4}{3} \frac{\alpha}{e} \log \frac{\mathrm{N}_{a}}{\mathrm{~N}_{b}} \mathrm{~T} \pm \sigma_{1} \Delta t\right)}{\Delta t\left(\frac{k_{1}}{p_{1}}+\frac{k_{2}}{p_{2}}\right)} \\
& =\frac{\frac{3}{e} \frac{\alpha}{\log } \mathrm{N}_{a}\left(\frac{4}{3} \frac{\alpha}{e} \log \frac{\mathrm{N}_{a}}{\mathrm{~N}_{b}} \mathrm{~T}_{1} \pm \sigma_{1} \Delta t\right)}{\frac{k_{1}}{p_{1}}+\frac{k_{2}}{p_{2}}} .
\end{aligned}
$$

In the stationary state $\Delta t=0$ and we get

$$
\omega^{\prime}=\frac{\mathrm{T}_{1}\left(\frac{4}{3} \frac{\alpha}{e} \log \frac{\mathrm{N}_{a}}{\mathrm{~N}_{b}}\right)^{2}}{\frac{k_{1}}{p_{1}}+\frac{k_{2}}{p_{2}}} .
$$

This false resistance superposed on the other resistance makes the electrical resistance of alloys greater than the value expected from the simple view of mixture. If we suppose the molecules of an alloy to be distributed in every direction in the same way, then only the third part of the doublets will oppose the current flowing in a certain direction. Therefore

$$
\omega^{\prime}=\frac{\mathrm{T}_{1}}{3} \frac{\left(\frac{4}{3} \frac{a}{e} \log \frac{\mathrm{N}_{a}}{\mathrm{~N}_{b}}\right)^{2}}{\frac{k_{1}}{p_{1}}+\frac{k_{2}}{p_{2}}} .
$$

Let $p_{1}$ be so small in comparison with $p_{2}$ that we may neglect $\frac{k_{2}}{p_{2}}$ beside $\frac{k_{1}}{p_{1}}$.

We get

$$
\omega^{\prime}=\frac{1}{3} \mathrm{~T}_{1}\left(\frac{4}{3} \frac{\alpha}{e} \log \frac{\mathrm{N}_{a}}{\mathrm{~N}_{b}}\right)^{2} \frac{p_{1}}{k_{1}}
$$

The false additional resistance is a reversible proportional to the thermal conductivity of the impurity. Since

$$
\mathrm{Q}=\frac{4}{3} \frac{\alpha}{e} \mathrm{~T}_{1} \log \frac{\mathrm{N}_{a}}{\mathrm{~N}_{b}}
$$


we get

$$
\omega^{\prime}=\frac{1}{3 T_{1}}\left(Q_{1}\right)^{2} \frac{p_{1}}{k_{1}} ;
$$

in the general case:

$$
\omega^{\prime}=\frac{1}{3 \mathrm{~T}_{1}} \frac{\mathrm{Q}_{11}{ }^{2}}{\frac{k_{1}}{p_{1}}+\frac{k_{2}}{p_{2}}} .
$$

Let us take the metals copper and zinc. The thermal conductivity of copper $k_{2}=0.94 \mathrm{~cm}$. gr. sec. cal.; the thermal conductivity of zinc $k_{1}=0 \cdot 26$. Let us consider an alloy of these two metals containing $40 \mathrm{gr}$. zinc and $60 \mathrm{gr}$. copper. The density of copper being $8 \cdot 8$, that of zinc $7 \cdot 1$, we shall have $45 \mathrm{~cm}^{3}$ zinc and $55 \mathrm{~cm} .^{3}$ copper, therefore

$$
\begin{aligned}
& p_{1}=0.45, \quad p_{2}=0.55, \\
& \frac{k_{1}}{p_{1}}=\frac{0 \cdot 26}{0 \cdot 45}=0.58, \quad \frac{k_{2}}{p_{2}}=\frac{0.94}{0.55}=1.71, \\
& \frac{k_{1}}{p_{1}}+\frac{k_{2}}{p_{2}}=2 \cdot 29 .
\end{aligned}
$$

The Peltier effect between copper and zinc is

$$
\frac{5 \cdot 85}{36.10^{2}}=1 \cdot 62 \cdot 10^{-3}=Q_{1} ; Q_{1}{ }^{2}=2 \cdot 62 \cdot 10^{-6},
$$

the false resistance at $0^{\circ} \mathrm{C}$. would be

$$
\begin{aligned}
& \omega^{\prime}=\frac{2 \cdot 62 \cdot 10^{-6}}{3 \cdot 273 \cdot 2 \cdot 29}=1 \cdot 4 \cdot 10^{-9} \cdot 4 \cdot 2 \cdot 10^{7}, \\
& \omega^{\prime}=5 \cdot 9 \cdot 10^{-2} \text { absolute unit. } \\
& \omega^{\prime}=5 \cdot 9 \cdot 10^{-11} \mathrm{ohm} .
\end{aligned}
$$

The electric conductivity of copper is $60.10^{4}$, its specific resistance $\frac{1}{60} \cdot 10^{-4}=1 \cdot 6 \cdot 10^{-6}$; the electric conductivity of zinc is $16.10^{4}$ per $\mathrm{cm}^{3}$, its specific resistance

$$
\frac{1}{16} \cdot 10^{-4}=6 \cdot 2 \cdot 10^{-6}
$$

If the resistance of the alloy copper and zine were to be found as the property of a simple mixture, we should get:

$$
\frac{(1 \cdot 6.55+45.6 \cdot 2)}{100} \cdot 10^{6}=3 \cdot 67.10^{-6} \text {. }
$$


788 Corpuscular Theory of the Thermoelectric Forces.

The actual electric conductivity of the alloy considered is equal to $13 \cdot 58 \cdot 10^{4}$, its specific resistance therefore $7 \cdot 4 \cdot 10^{-6}$; thus, the actual resistance is very nearly twice as large as that calculated from the mixture formula. The false resistance $\omega^{\prime}=5^{\cdot 5} \cdot 10^{-11}$ is exceedingly small in comparison with the difference between the actual and the calculated resistance.

We shall now consider an alloy made up of $99 \cdot 3 \mathrm{gr}$. copper and $0.7 \mathrm{gr}$. zinc. Using the same notation as before we get

$$
\begin{aligned}
p_{1} & =0 \cdot 01, \quad \frac{k_{1}}{p_{1}}+\frac{k_{2}}{p_{2}}=26+0 \cdot 95, \\
p_{2} & =0 \cdot 99, \quad \\
\omega^{\prime} & =\frac{1}{3.273} \cdot \frac{2 \cdot 62 \cdot 10^{-6}}{26 \cdot 95}=1 \cdot 19 \cdot 10^{-10}, \\
\omega^{\prime} & =1 \cdot 19 \cdot 10^{-10} \cdot 4 \cdot 2 \cdot 10^{7}, \\
\omega^{\prime} & =5 \cdot 10^{-3} \text { absolute unit, } \\
\omega^{\prime} & =5 \cdot 10^{-3} \cdot 10^{-9}=5 \cdot 10^{-12} \text { ohm. }
\end{aligned}
$$

The mean resistance would be:

$$
10^{-6}(1 \cdot 6.0 \cdot 99+6 \cdot 2 \cdot 0 \cdot 01)=1 \cdot 64 \cdot 10^{-6} \text {. }
$$

But the actual conductivity is equal to $54 \cdot 56 \cdot 10^{4}$; therefore the actual specific resistance is equal to $1 \cdot 84 \cdot 10^{-6}$. Here again the actual resistance is found to be larger than the calculated one, by an amount of quite a different order of magnitude from the false resistance.

The last example may be an alloy composed of equal volumes of copper and platinum. The thermal conductivity of platinum $k_{1}$ is equal to $0 \cdot 17$, the electric conductivity $9 \cdot 2 \cdot 10^{4}$, the specific resistance $10 \cdot 8 \cdot 10^{-6}$. The Peltier effect between the two metals is equal to $Q_{1}=8 \cdot 9 \cdot 10^{-4}$.

$$
\begin{aligned}
& \mathrm{Q}_{1}^{2}=79 \cdot 10^{-8} \\
& \frac{k_{1}}{p_{1}}+\frac{k_{2}}{p_{2}}=0 \cdot 17 \cdot 2+0 \cdot 94.2=2 \cdot 22 .
\end{aligned}
$$

The false resistance would be equal to

$$
\begin{aligned}
& \omega^{\prime}=\frac{1}{3} \cdot \frac{79 \cdot 10^{-8}}{3 \cdot 273 \cdot 2 \cdot 22}=4 \cdot 35 \cdot 10^{-10}, \\
& \omega^{\prime}=4 \cdot 35 \cdot 10^{-10} \cdot 4 \cdot 2 \cdot 10^{7}=1 \cdot 82 \cdot 10^{-2}, \\
& \omega^{\prime}=1 \cdot 82 \cdot 10^{-11} \mathrm{ohm} .
\end{aligned}
$$

In this case we shall calculate this false resistance from the first formula given by Lord Rayleigh:

$$
\omega^{\prime}=273 e^{2}\left(\frac{k_{1}}{p_{1}}+\frac{k_{2}}{p_{2}}\right)^{-1},
$$


$e$ is the thermoelectric force of the element copper-platinum for a difference of temperature of 1 degree.

$$
\begin{aligned}
& e=0 \cdot 72 \cdot 10^{-5} \cdot 10^{8} \text { absolute units. } \\
& e^{2}=0 \cdot 52 \cdot 10^{6} \\
& \omega^{\prime}=\frac{273 \cdot 0 \cdot 52 \cdot 10^{6}}{2 \cdot 22 \cdot 4 \cdot 2 \cdot 10^{7}}=15 \cdot 2 \cdot 10^{-1} \text { absolute unit, } \\
& \omega^{\prime}=1 \cdot 52 \cdot 10^{-9} \mathrm{ohm} .
\end{aligned}
$$

The mean electric conductivity of the alloy is found to be* $3 \cdot 09.10^{4}$; the specific electric resistance would be

$$
\omega=0 \cdot 323 \cdot 10^{-4} \text {. }
$$

Considering the alloy as a single mixture we should find

$$
\frac{1}{2}(10 \cdot 8+1 \cdot 6) \cdot 10^{-6}=0 \cdot 062 \cdot 10^{-4}
$$

The false resistance is in these cases far too small to explain the difference between the observed and the calculated value of the resistance of the alloys. This result may partly explain why Dr. R. S. Willows $†$ could detect no difference between the apparent resistances of alloys with direct current and with alternating current.

An alloy of equal volumes of copper and iron has a false resistance amounting to 1.5 per cent. of that of copper.

Cambridge, April 6th, 1908.

LXVI. A Recalculation of the Vapour Pressure of Mercury. By T. H. LABY, B.A., Exhibition of 1851 Research Scholar, Joule Student of the Royal Spciety, Emmanuel College, Cambridge

7 WE vapour-pressure of mercury is intrinsically important: 1 it has been determined for a wider range of temperatures than that of any other substance ; and Professor Nernst hopes to be able to find the integration constant of the equation of the reaction isochore "by continuing the vapour-pressure curves as far as possible in the direction of very small pressures, for example to $001 \mathrm{~mm}$." $\S$ Yet the greatest-and it should be added, unnecessary - disagreement is to be found in the current values of this vapour-pressure, nor is there any table

* Landolt-Börnstein Physikalish-chemische Tabellen, 3 Auflage, p. 721a.

+ R. S. Willows, 'Proceedings of the Physical Society of London,' vol. xx. p. 427 (1907).

I Communicated by the Author.

$\$$ Nernst, 'Thermodynamics and Chemistry,' New York, 1907, p. 60. 